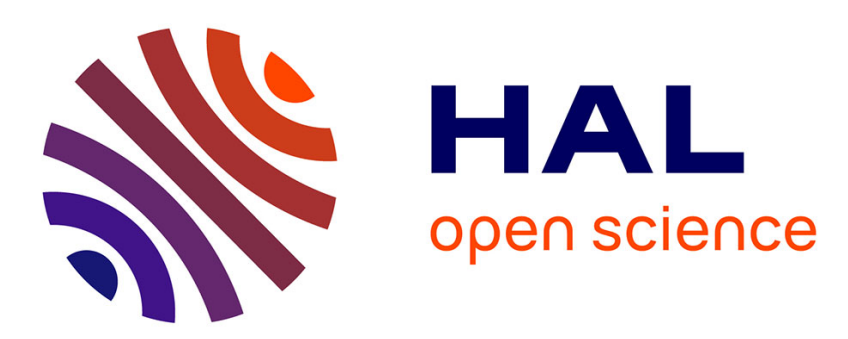

\title{
Columnar dendritic solidification of TiAl under diffusive and hypergravity conditions investigated by phase-field simulations
}

\author{
A Viardin, Julien Zollinger, L. Sturz, M Apel, J. Eiken, R. Berger, U. Hecht
}

\section{To cite this version:}

A Viardin, Julien Zollinger, L. Sturz, M Apel, J. Eiken, et al.. Columnar dendritic solidification of TiAl under diffusive and hypergravity conditions investigated by phase-field simulations. Computational Materials Science, 2020, 172, pp.109358. 10.1016/j.commatsci.2019.109358 hal-02751963

\section{HAL Id: hal-02751963 \\ https://hal.science/hal-02751963}

Submitted on 3 Jun 2020

HAL is a multi-disciplinary open access archive for the deposit and dissemination of scientific research documents, whether they are published or not. The documents may come from teaching and research institutions in France or abroad, or from public or private research centers.
L'archive ouverte pluridisciplinaire HAL, est destinée au dépôt et à la diffusion de documents scientifiques de niveau recherche, publiés ou non, émanant des établissements d'enseignement et de recherche français ou étrangers, des laboratoires publics ou privés. 


\title{
Columnar dendritic solidification of TiAl under diffusive and hypergravity conditions investigated by phase-field simulations
}

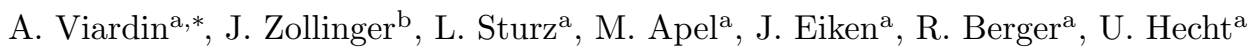 \\ ${ }^{a}$ ACCESS e.V., Intzestrasse 5, D-52072 Aachen, Germany. \\ ${ }^{b}$ Institut Jean Lamour, CNRS - Université de Lorraine, ARTEM Campus - \\ Allée André Guinier, F-54000 Nancy, France.
}

\begin{abstract}
Based on 2D phase-field simulations including fluid flow driven by natural convection, columnar dendritic growth of the $\beta$-solidifying Ti- 48 at\% $\mathrm{Al}$ alloy is characterised for different gravity levels ranging from 0 to $\pm 15 \mathrm{~g}$. Depending on the direction of the gravity $g$ with respect to the growth direction, different flow regimes emerge which show stable or unstable dendritic growth dynamics. When gravity and growth directions are parallel, the dendrite tips experience downward melt flow and individual dendrites grow in a stable manner with a rather small modification of the operating state. When gravity and growth directions are antiparallel, the impact on the operating state is larger. Eventually, at higher gravity levels the upward melt flow around the dendrite tips "destabilises" the dendritic morphology resulting in tip splitting, branching and local changes in the apparent dendrite growth direction which is an alternative mechanism for the adjustment of the primary dendrite arm spacing in addition to tertiary arm formation.
\end{abstract}

Keywords: Phase-field, fluid flow, solidification, hypergravity, microstructure

\section{Introduction}

Dendritic growth during solidification is governed by the interplay between diffusion of heat and solute, capillarity and melt flow which is often neglected due to its complexity. Consequently, experiments have been designed to suppress natural convection under microgravity conditions [1] or by using thin samples [2]. Dendritic growth under pure diffusive conditions has been experimentally and numerically studied [3-7]. The effect of natural convection on dendritic growth was investigated in experiments $[8,9]$ and simulations [10-12]. Gravity effects on the solidification behavior were simulated for an $\mathrm{Al}-\mathrm{Cu}$ alloy [13-15] and analyzed experimentally for a $\mathrm{Pb}-\mathrm{Sn}$ alloy [16]. The authors observed that depending on the direction and magnitude of the gravity vector, the gravity induced natural solutal convection affects the primary dendrite arm spacing PDAS. At present, our understanding of the interaction between melt flow and solidification patterns under different gravity conditions is still incomplete, despite its relevance for the microstructure formation, e.g. in centrifugal casting. Several newly developed investment casting technologies make use of moderated to high centrifugal forces to promote mold filling in thin parts [17]. In particular, titanium aluminide structural parts are produced with centrifugal casting processes, both for automotive and aircraft applications. Herein, accelerations can be as high as $20 g[18,19]$.

\footnotetext{
*Corresponding author
}

In this paper, we present and discuss 2D phase-field simulations of columnar dendritic solidification of the bcc $\beta(\mathrm{Ti})$ phase of the binary alloy $\mathrm{Ti}-48 \mathrm{at} \% \mathrm{Al}$ for various directions and magnitudes of the gravity vector. The simulations substantiate and complement the analysis of experimental work for Ti-47.5Al-2Cr-2Nb (all at\%) solidification under microgravity and hypergravity conditions.

The paper is organized as follows: In section 2 the experimental set-up and the most important microstructural observations are briefly outlined. In section 3 , the modelling approach and parameters are described. In section 4, the simulations results are presented and discussed, mainly regarding the effect of convective flow on the dendrite morphology. Finally the results are summarized in section 5 and outline the most important conclusions.

\section{Brief overview of experiments and the impact of gravity on PDAS in Ti-47.5Al-2Cr-2Nb}

The full details of the directional solidification experiments performed in micro- and hyper-gravity and its modelling at the macro-scale will be presented in a separate paper [20]. Only the results relevant to dendritic growth morphologies discussed in this paper are presented in the following.

\subsection{Materials and Methods}

A $4 \mathrm{~kg}$ ingot (70 $\mathrm{mm}$ in diameter and $250 \mathrm{~mm}$ in height) of Ti-47.5Al-2Cr-2Nb GE alloy was provided by Safran Aircraft Engines. Samples for directional solidification 
were spark eroded to produce cylinders with $8 \mathrm{~mm}$ diameter and $200 \mathrm{~mm}$ length. The furnace for directional solidification is a gradient zone furnace in which the constrained crystal growth is achieved using a "power-down" technique and controlled using three thermocouples located on the three heaters of the furnace and one thermocouple in contact with the sample. Directional solidification experiments in hyper-gravity conditions were conducted in the Large Diameter Centrifuge (LDC) of the European Space Agency Technical Center in Noordwijk aan Zee, Netherlands [21]. In the experiments, the acceleration forces are opposite to the growth direction; such configuration will be noted $-g$ in the following. Five acceleration levels corresponding to $-1,-5,-10,-15$ and $-20 g$ were selected. Microgravity experiments were carried out in the same furnace aboard the MAXUS 9 sounding rocket on April 7th, 2017. The samples processed in micro- and hyper-gravity have undergone the same thermal cycle and the only variable of the experimental protocol is the gravity level.

\subsection{Microstructure evolution with gravity}

Figure 1 shows SEM-BSE images of a transverse section for the samples solidified in micro-gravity $(\mu g)$ and in hyper-gravity conditions $(-18 g)$. A significant reduction of the average PDAS $\lambda_{a v}$ is observed, from $\lambda_{a v} \approx 800 \mu \mathrm{m}$ under $\mu g$ to $\lambda_{\mathrm{av}} \approx 400 \mu \mathrm{m}$ under $-18 \mathrm{~g}$ conditions. The reason for the reduction of $\lambda_{a v}$ is melt flow in the mushy zone mainly associated with the segregation of the lighter element aluminum. A quantitative relation between the PDAS and the gravity level will be presented in a dedicated paper along with the observed scaling law. Here, it is sufficient to note that the system's response to flow is qualitatively similar to the one described by Bataille [16] for $\mathrm{Pb}-50 \mathrm{wt} \% \mathrm{Sn}$. The PbSn- and TiAl-alloys will likely differ with respect to the mechanisms behind spacing selection, e.g. by tertiary branching and/or tip splitting [22]. From phase-field modelling, we expected a better insight into the selection mechanisms, in particular about the dynamics and stability of dendrite tips.

\section{Modeling}

\subsection{Phase-field model}

For the phase-field simulations, the MICRESS [23] software was used. The implemented phase-field model is based on the multiphase-field concept [24] and includes multicomponent diffusion. However, it reduces to a single phase-field, binary alloy model in the present study. The numerical implementation respects the thin interface analysis [25, 26] and comprises a finite-difference correction to improve the numerical accuracy of the results [27] and to allow computations with a low number of interface grid points. The melt flow is simulated by solving the incompressible Navier-Stokes equations in a finite difference formulation. A no-slip condition for the melt flow at the diffuse solid-liquid interface is modeled as a friction term

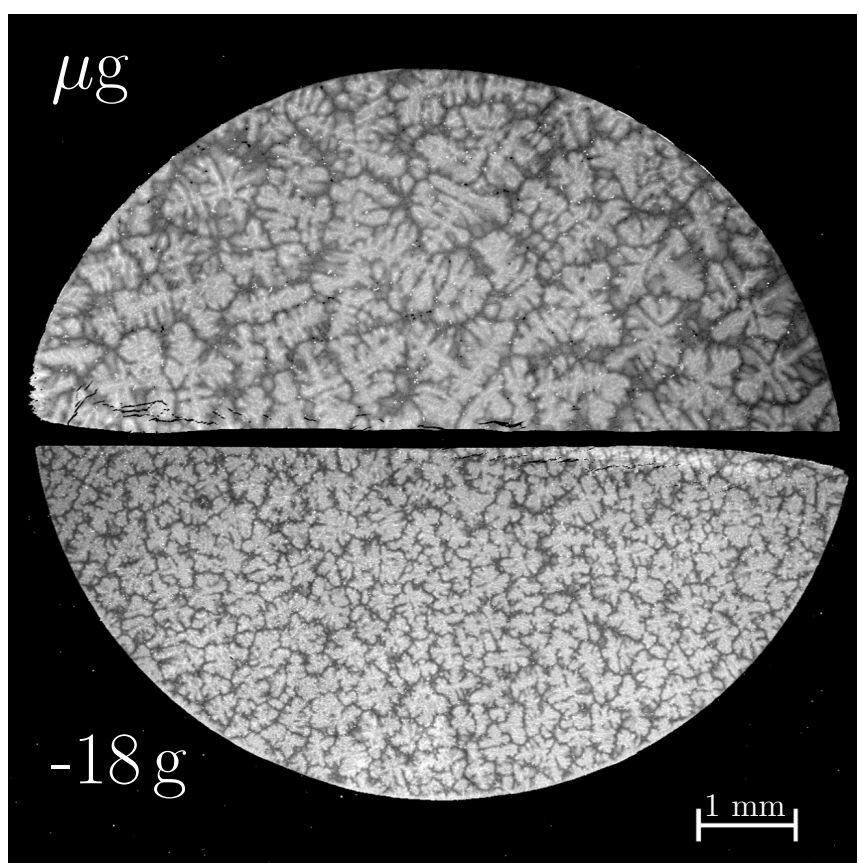

Figure 1: SEM-BSE image of the columnar dendritic structure in the transverse section for a $\mathrm{Ti}-47.5 \mathrm{Al}-2 \mathrm{Cr}-2 \mathrm{Nb}$ alloy processed in microgravity $(\mu g)$ and in hypergravity conditions $(-18 g)$, respectively.

in the diffuse interface region as described in [13, 28]. To model buoyancy forces the Boussinesq approximation is used. The partial differential equations for flow and pressure are solved on a grid with twice the spacing used for the concentration field and phase-field (where the grid size is $\Delta \mathrm{x}=\Delta \mathrm{z}=0.75 \mu \mathrm{m}$ ), a method suggested by Lu et al. [29]. In order to calculate the advection term, the flow velocity is projected on the finer grid using linear interpolation. The coarser grid for the flow field leads to a computational speed up by a factor of five for the simulations shown in this paper.

\subsection{Simulation parameters}

The Ti-47.5Al-2Cr-2Nb alloy used in the experiments is approximated for the simulations by a binary $\mathrm{Ti}-48 \mathrm{Al}$ alloy which has a similar pro-peritectic phase fraction. In Table 1 the Ti-48 at\%Al alloy properties used for the calculations are given. For the binary alloy a linearised phase diagram approximation defined by the liquidus slope and partition coefficient is sufficient. Only the density change by the local composition was accounted in the simulations, i.e. the temperature dependency of the melt density is neglected.

The flow pattern is expected to be affected by the geometry of the calculation domain. To get an idea about this influence, simulations are performed in a rectangular domain of $450 \times 1050 \mu \mathrm{m}^{2}$ and $900 \times 1050 \mu \mathrm{m}^{2}$. Initially, a spherical seed is set at the bottom center. The general coordinate system is defined in Figure 2. The boundary conditions are set to be periodic in $x$-direction for concentra- 
tion, phase-field, pressure and flow. A fixed concentration of $48 \mathrm{at} \% \mathrm{Al}$ is set on the top boundary, for pressure and velocity a zero gradient condition is set on the top and bottom boundaries. The computational domain moves with the dendrite tips (i.e. moving frame calculations) at a constant distance of $450 \mu \mathrm{m}$ between the most advanced tip and the top boundary. The thermal parameters for modelling directional solidification in the so called "frozen temperature approximation" are a constant temperature gradient in $\mathrm{z}$-direction of $120 \mathrm{~K} \mathrm{~cm}^{-1}$ and a constant cooling rate of $-0.3 \mathrm{~K} \mathrm{~s}^{-1}$ which corresponds to a pulling velocity of $\mathrm{V}_{\text {pull }}=25 \mu \mathrm{m} \mathrm{s}^{-1}$. An interface thickness of $3 \mu \mathrm{m}$ and a phase-field mobility of $\mu_{s l}=10^{-2} \mathrm{~cm}^{4} \mathrm{~J}^{-1} \mathrm{~s}^{-1}$ were selected by calibration of these parameters under purely diffusive conditions and steady state growth conditions according to the thin-interface limit. The flow pattern in a $2 \mathrm{D}$ calculation domain is qualitatively different compare to $3 \mathrm{D}$ flow as shown for equiaxed case by [30, 31]. Thus, our results will be different compared to experimental 3D flow scenario but they demonstrate basic principles concerning the interaction between flow and tip operating state. The boundary conditions are not fully representative for the experimental case, especially the conditions at the top boundary does not represent a link to the far-field melt flow established at the macro-scale [32] of the whole melt volume. Keeping this limitation and the confinement to $2 \mathrm{D}$ in mind, one may not expect a quantitative match to experimental results. Therefore, the aim of the simulations is rather a fundamental study of the interaction between columnar growing dendrites with solutal driven convection, induced by their own segregation, under systematically varied gravity conditions in a generic set-up.

\section{Results and discussion}

\subsection{Effect of gravity level on $\beta(T i)$ dendrite growth}

The results of $2 \mathrm{D}$ phase-field simulations of $\beta(\mathrm{Ti})$ dendrite growth show the different dendritic morphologies in the mushy zone according to the direction and magnitude of the gravity vector. Figures 2 and 3 show spatiotemporal plots of the solid-liquid interface contours (at a solid fraction $f_{s}=0.5$ ) for a domain width of $W=450 \mu \mathrm{m}$ and $W=900 \mu \mathrm{m}$, respectively. For the case of $W=450 \mu \mathrm{m}$, the microstructure remains dendritic and stable for positive and low negative gravity levels (from $-3 \mathrm{~g}$ to $15 \mathrm{~g}$ ). Compared to the pure diffusive case $(0 g)$, the tip position is more advanced for positive gravity and falls behind for negative gravity levels, i.e. the steady state tip temperature varies with the gravity level. At $-4 g$, a transition between dendritic and branched structures is observed. For $-20 \mathrm{~g}$ to $-5 \mathrm{~g}$, the microstructure is branched with an decreasing number of splitting events. At $-5 g$ the microstructure and splitting events are periodic while they seem to be random between $-10 \mathrm{~g}$ and $-20 \mathrm{~g}$. In Figure 3, similar observations can be made for the wider domain, but the transition from dendritic to branched morphology has already occurred at $-1 g$. The cases at $-4 g$ for
$W=450 \mu \mathrm{m}$ and $-1 \mathrm{~g}$ at $W=900 \mu \mathrm{m}$ show splitting events during early stages of growth, while they reached stable dendritic growth afterwards.

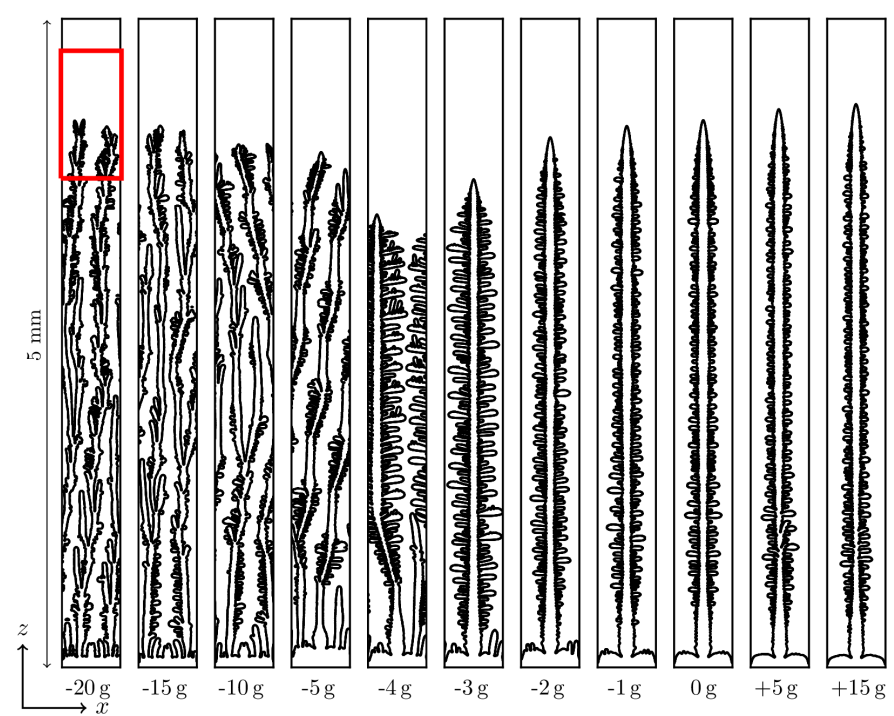

Figure 2: Spatiotemporal plots of the solid/liquid boundary for different gravity levels after $200 \mathrm{~s}$. Domain width is $450 \mu \mathrm{m}$. A positive gravity vector is oriented in the same direction as the growth direction, a negative gravity vector in the opposite direction. The red rectangle represents the moving calculation domain. Please note that the interface does not move further outside the moving calculation domain.

The selected dendritic growth structures are correlated with the melt flow pattern. In Figure 4 concentration maps are superimposed to flow streamlines at different gravity levels and times for the domain width $W=450 \mu \mathrm{m}$. After $50 \mathrm{~s}$, steady state is not yet reached for all gravity levels, but several features can be observed already. For all positive gravity levels, the convection rolls are largely symmetric with respect to the dendrite axis and the dendrite tips are opposed to a downward flow that transports "bulk liquid" to the tip which has a lower Al-composition compared to the tip composition, thus reducing the solutal undercooling. In this case, the dendrite tip has favorable growth conditions [13]. For gravity levels ranging from $-3 g$ to $-1 g$, the central dendrite remains dominant, with symmetric convection rolls but with an upward flow at the dendrite tip. Upward flow transports supersaturated liquid from the interdendritic region to the dendrite tip, but also transports the Al-rich liquid away from the tip towards the melt volume. In this case, the flow destabilizes the dendritic growth pattern. For $-15 \mathrm{~g}$ and $-10 \mathrm{~g}$, a change of the PDAS by branching can be observed in qualitative agreement to the experimental result given in Figure 1.

The PDAS selection for $-10 \mathrm{~g}$ at an early stage between $30 \mathrm{~s}$ and $50 \mathrm{~s}$ is shown in Figure 5 . At $30 \mathrm{~s}$, one can see an upward flow at the central dendrite tip and downward flow at the sides. From $30 \mathrm{~s}$ to $45 \mathrm{~s}$, the dendrites on the side are growing faster than the central one because of favorised growth conditions induced by downward flow. Between 
Density (liquid) $(\rho)$

Density variation $(\partial \rho / \partial c)$

Kinematic viscosity $(\nu)$

Liquidus slope $\left(m_{l}\right)$

Partition coefficient $\left(k_{A l}\right)$

Diffusion coefficient in the melt $\left(D_{l}\right)$

Interfacial energy $\left(\sigma_{s l}\right)$

Interfacial energy anisotropy $\left(\epsilon_{s l}^{\sigma}\right)$

Transformation entropy density $\left(\Delta S^{m}\right)$

$3.7 \cdot 10^{6}$
$-1.77 \cdot 10^{4}$
$1.89 \cdot 10^{-6}$
-11.268
0.9
$3 \cdot 10^{-9}$
0.1
$1.1 \%$
0.62

$\mathrm{g} \mathrm{m}^{-3}$
$\mathrm{~g} \mathrm{~m}^{-3} \mathrm{at}^{2} \mathrm{Al}^{-1}$
$\mathrm{~m}^{2} \mathrm{~s}^{-1}$
$\mathrm{Kat} \%^{-1}$
-
$\mathrm{m}^{2} \mathrm{~s}^{-1}$
$\mathrm{~J} \mathrm{~m}^{-2}$
-
$\mathrm{Jcm}^{-3} \mathrm{~K}^{-1}$

$[45]$

45

$[46]$

$[47]$

$[48]$

49

49]

[49]

Table 1: Physical and numerical parameters used in the simulations.
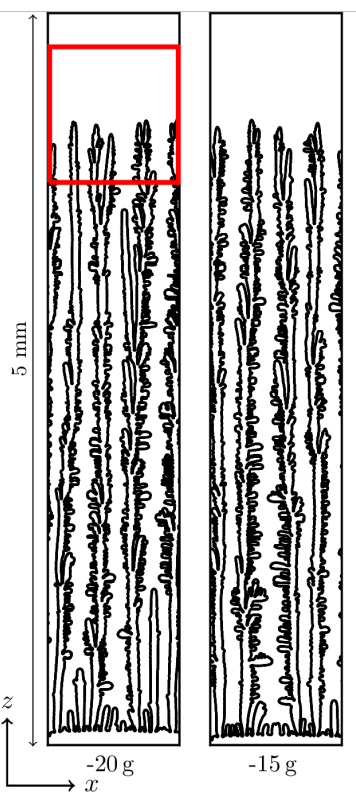

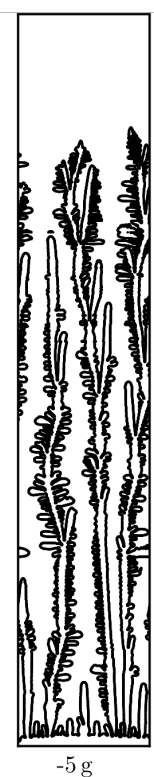

$-5 \mathrm{~g}$

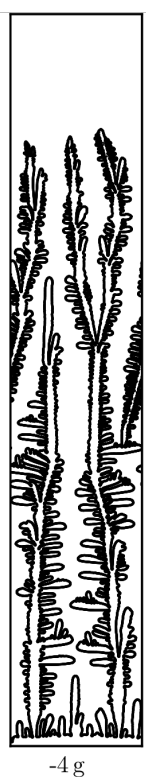

$-4 \mathrm{~g}$

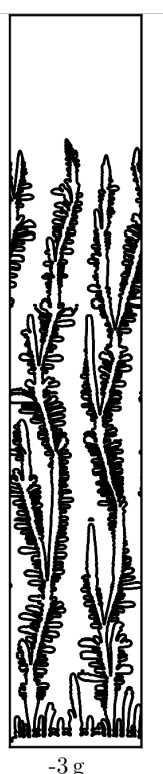

$-3 \mathrm{~g}$
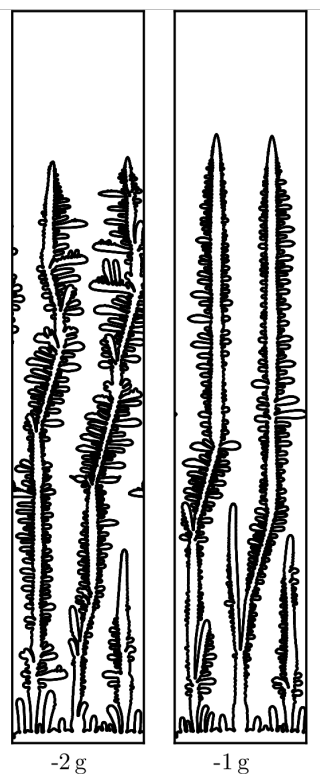
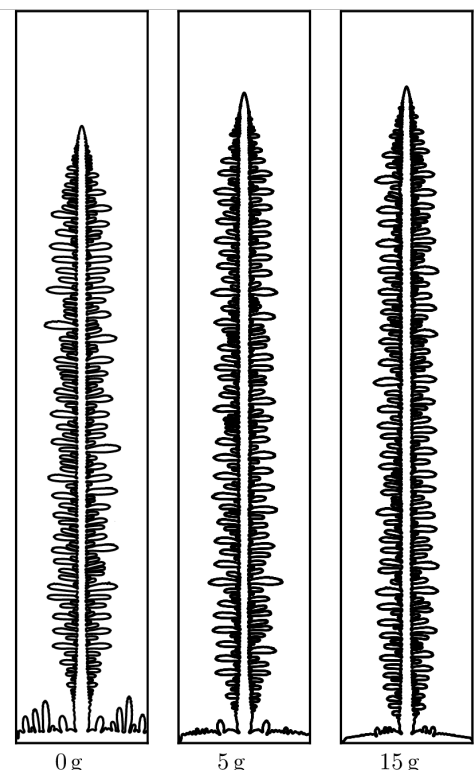

$15 \mathrm{~g}$

Figure 3: Spatiotemporal plots of the solid/liquid boundary for different gravity levels after $200 \mathrm{~s}$. Domain width is $900 \mu \mathrm{m}$. A positive gravity vector is oriented in the same direction as the growth direction, a negative gravity vector in the opposite direction. The red rectangle represents the moving calculation domain. Please note that the interface does not move further outside the moving calculation domain.

$45 \mathrm{~s}$ and $50 \mathrm{~s}$, the PDAS is about to change as well as the flow pattern, dendrites on the sides experience upward flow, the central dendrite splits at the tip. One can also remark that the distances between concentration isolines in the tip region are smaller when the tips are exposed to a downward flow compared to those within an upward flow.

At times $\mathrm{t} \geq 50 \mathrm{~s}$, the microstructure remains dendritic with a single dendrite for gravity levels between $-3 g$ and $+15 \mathrm{~g}$. Furthermore, when the gravity level is between $-15 \mathrm{~g}$ and $-5 \mathrm{~g}$, a succession of splitting events can be observed which leads to a decreasing PDAS. The convection rolls evolves dynamically over time.

To characterize the velocity fields in Figure 4 by a single number, we have applied the following procedure: first we averaged the flow velocity in the $\mathrm{z}$-direction for every $\mathrm{x}$ position, then we determined the maximum of all averaged values $V_{\text {flow }}^{\max }$. $V_{\text {flow }}^{\max }$ as a function of the gravity level is plotted in Figure 6(a) for both domain widths. For positive gravity levels, flow velocities are negative at the tip, as was show in Figure 4. For negative gravity values, upward and downward flows occur at the dendrite tips. Only the maximum value for the upward flow is shown in the figure, as the corresponding values for downward flow are the same. The absolute values of the flow velocities are lower when gravity is positive, i.e. for the case of a rather localised convection role, compared to identical negative values and the corresponding large scale convection pattern in the bulk melt. This can be seen directly in the concentration field (Figure 4), e.g. by the shape and position of the isoline. The domain size affects the melt flow velocity as well, for the wider domain the velocities are higher (both for negative and positive gravity levels). Stable dendritic growth occurs when the flow velocity is below or close to the pulling velocity. Branched morphologies occur when the maximum positive velocities are above twice the pulling velocity. In between these two cases growth remains dendritic, i.e. at $-4 \mathrm{~g}$ for $W=450 \mu \mathrm{m}$ and at $-1 \mathrm{~g}$ at $W=900 \mu \mathrm{m}$, after only one splitting event.

Finally, the number of splitting events is shown in Figure 6(b) for all negative gravity levels, again for both domain widths. The number of splitting events $\mathrm{N}_{S}$ has been normalized by the number of primary arms $\mathrm{N}_{\mathrm{D}}$ to scale the results obtained for different domain widths. The behavior is similar for both domain widths and shows that the 


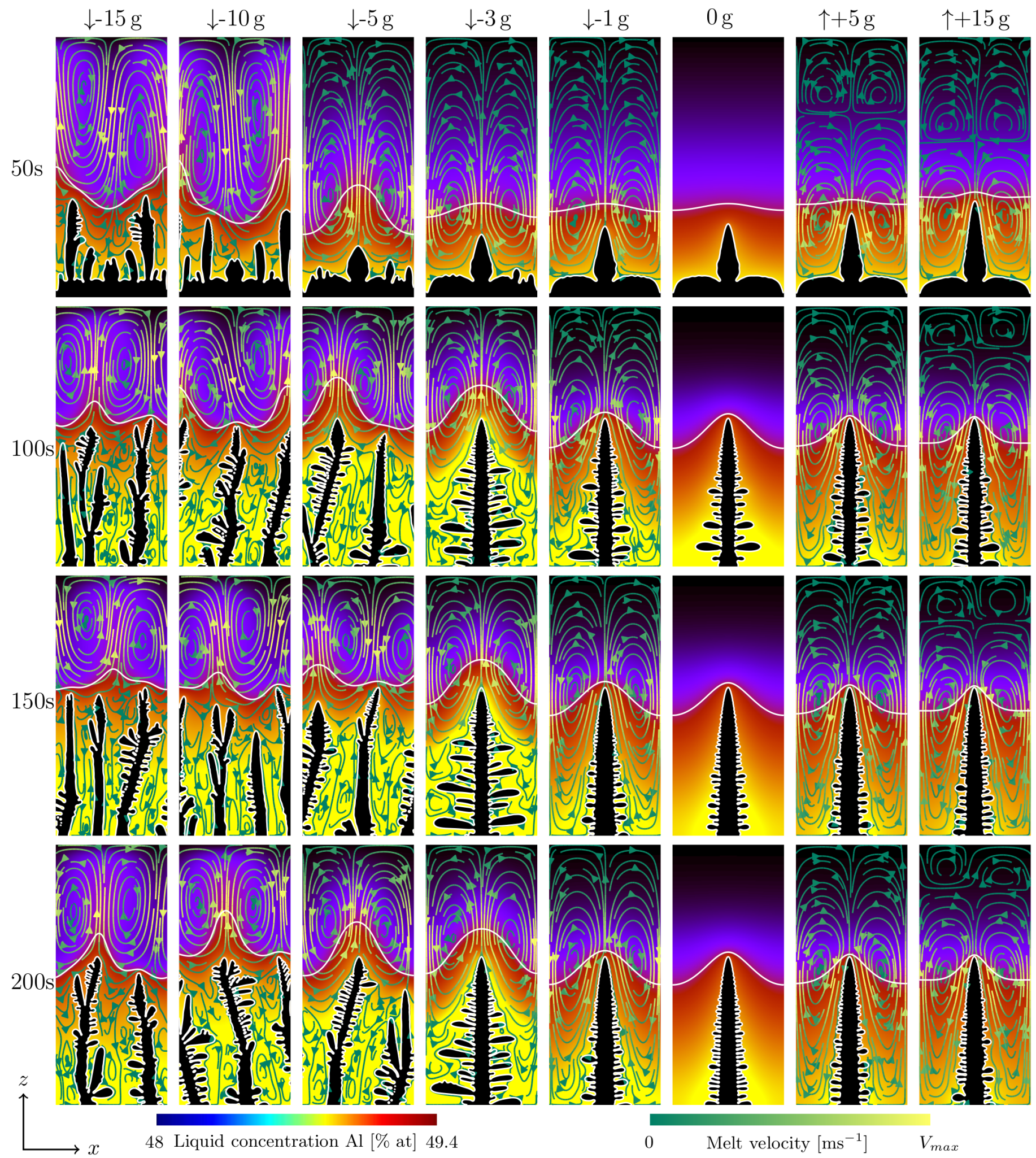

Figure 4: Dendrite growth in Ti-48at\%Al $(W=450 \mu \mathrm{m})$ as function of time and gravity level, concentration maps are superimposed with flow streamlines (the $V_{\max }$ value for flow is different for each figure), the white isoline corresponds to 48.6at\%Al. Please note that the color scale for the streamlines has been normalised to $V_{\max }$. 


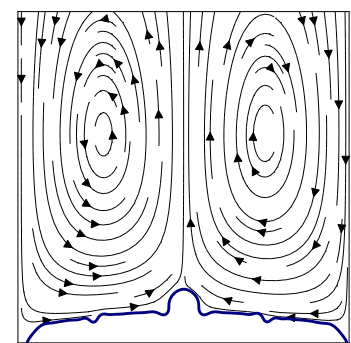

$30 \mathrm{~s}$

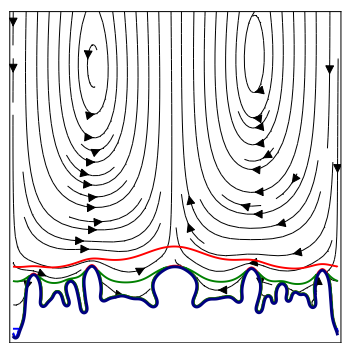

$35 \mathrm{~s}$

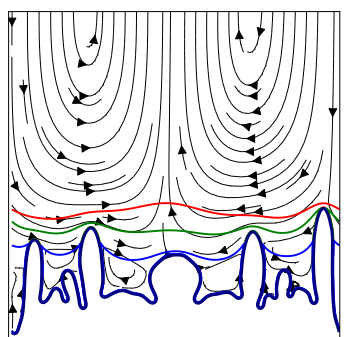

$40 \mathrm{~s}$

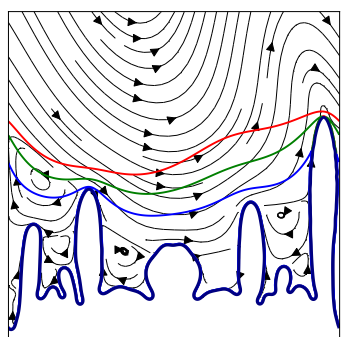

$45 \mathrm{~s}$

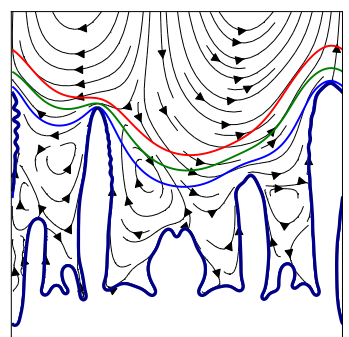

$50 \mathrm{~s}$

Figure 5: Initial evolution of the dendritic array at the bottom of the calculation domain superimposed with the flow pattern, the concentration isolines are given for $48.8,48.9,49 \mathrm{at} \% \mathrm{Al}$ with the red, green and blue isolines. Gravity level $=-10 \mathrm{~g}$ and $\mathrm{W}=450 \mu \mathrm{m}$. The selected cut out is $600 \times 600 \mu \mathrm{m}^{2}$.

number of splitting events increases with the magnitude of the gravity level. For $W=900 \mu \mathrm{m}$ splitting sets in already at $-1 g$, however this compares to $-5 g$ for the smaller domain which leads to similar flow velocities approximately twice the pulling velocity.

\subsection{Operating state of dendritic structures}

In this section, we discuss in more detail the change of the dendrite tip operating point under the influence of melt flow. As was shown in Figures 2 and 3, the dendrite tip position depends on the gravity level. The change of the tip position is linked to the dependency of the selection constant $\sigma^{*}$ from the flow velocity, which was investigated by Ben Ammar and Pomeau [33] using scaling laws. If the solutal boundary layer is thin compared to the hydrodynamic layer, they conclude that selection is possible for any amplitude of the flow and that the selection constant is the same as without flow. A more precise description of the selection mechanism with flow is given by Bouissou and Pelcé $[34,35]$. In their analytical study, surface tension is treated as a singular perturbation. The result of the calculation is that the selection mechanism is the same as in the absence of flow, i. e. the anisotropy of interface stiffness selects the tip radius $\rho$ and the tip velocity $V_{\text {tip }}$ according to the solvability criterion $\rho^{2} V_{\text {tip }}=$ const.

Bouissou et al. performed experiments on the evolution of $\rho$ and $V_{\text {tip }}$ with flow during dendritic growth in pivalic acid solution [36, 37] and ammonium bromide [37, 38], and made the following observations:

- the interface remains parabolic near the dendrite tip,

- $\rho^{2} V_{\text {tip }}$ is constant during growth in the presence of external flow,

- during columnar growth $\rho^{2} V_{\text {tip }}$ does not depend on the transverse component of the flow and increases linearly with the component of the flow parallel to the growth direction (i.e. the longitudinal component).

This relation between $\rho^{2} V_{\text {tip }}$ and longitudinal flow velocity is given by the empirical law (derived from analytical solutions in $[34,35])$ :
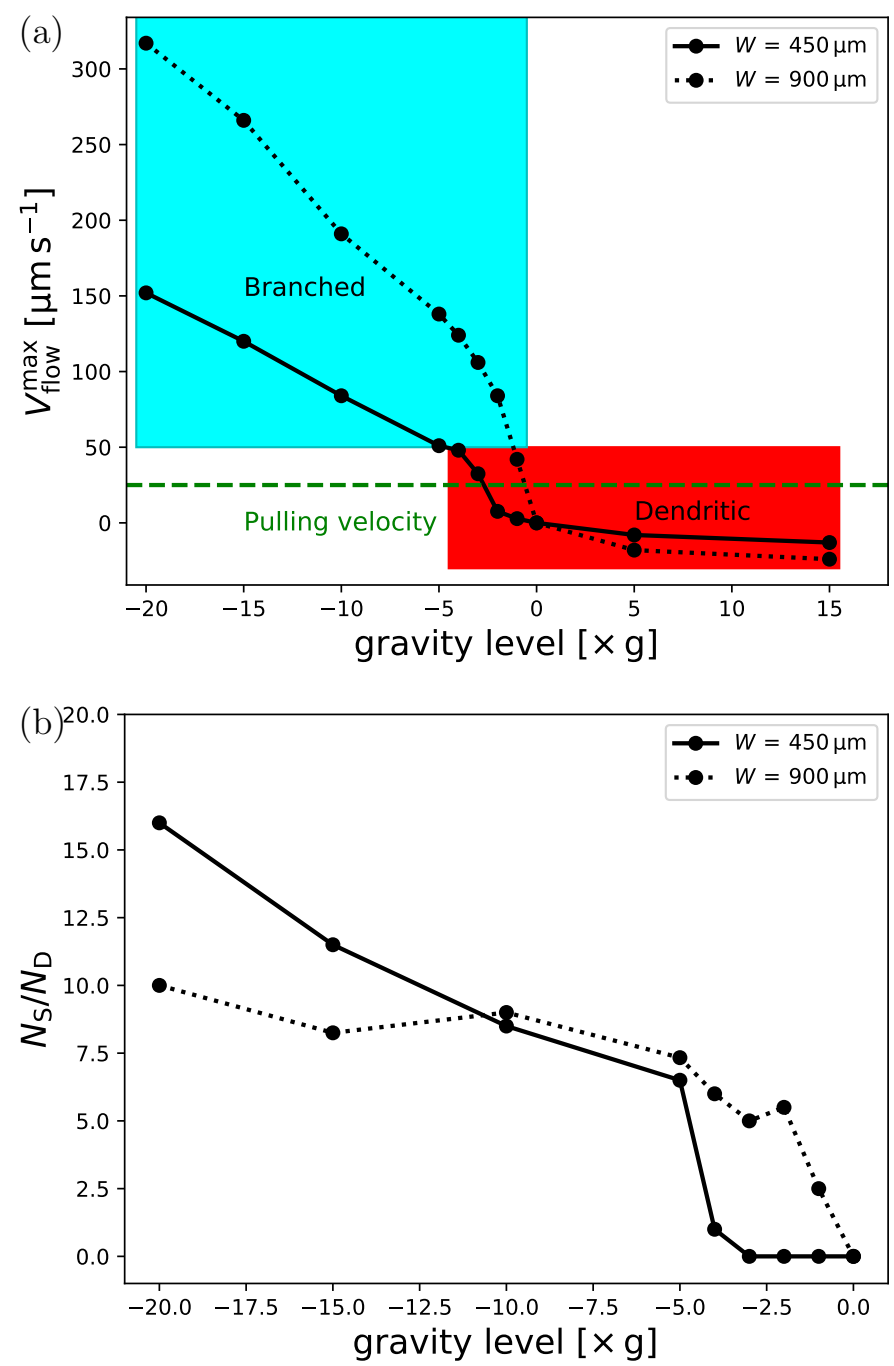

Figure 6: (a) Growth morphology and average $z$-flow velocity as a function of the gravity level. The pulling velocity at $25 \mu \mathrm{ms}^{-1}$ is represented by a green dashed line. (b) Number of splitting events divided by the number of primary arms as a function of gravity level for both domain widths. 


$$
\rho^{2} V_{\text {tip }}=\frac{C_{0}}{4} d_{0} D_{1}\left(1+\chi \frac{V_{\text {flow }}}{D_{1}} d_{0}\right)
$$

with

$$
C_{0}=4 \frac{\rho^{2} V_{\text {pull }}}{d_{0} D_{1}}
$$

where $d_{0}$ is the capillarity length, $D_{1}$ is the diffusion coefficient in the liquid, $V_{\text {flow }}$ and $V_{\text {pull }}$ are the flow and pulling velocities ( $V_{\text {flow }}$ is the averaged flow veloctiy measured in longitudinal z-direction), respectively, and the coefficient $\chi$ is depending on the interfacial energy anisotropy. The authors experimental measurements on pivalic acid results in values of $\chi=6400$ and $C_{0}=340$ [35]. Other experiments performed with succinonitrile by Lee et al. [39] could not be explained by equation (1), since $\rho^{2} V_{\text {tip }}$ was shown to decrease with flow velocity, which indicated possible disparity between chemical and thermal growth.

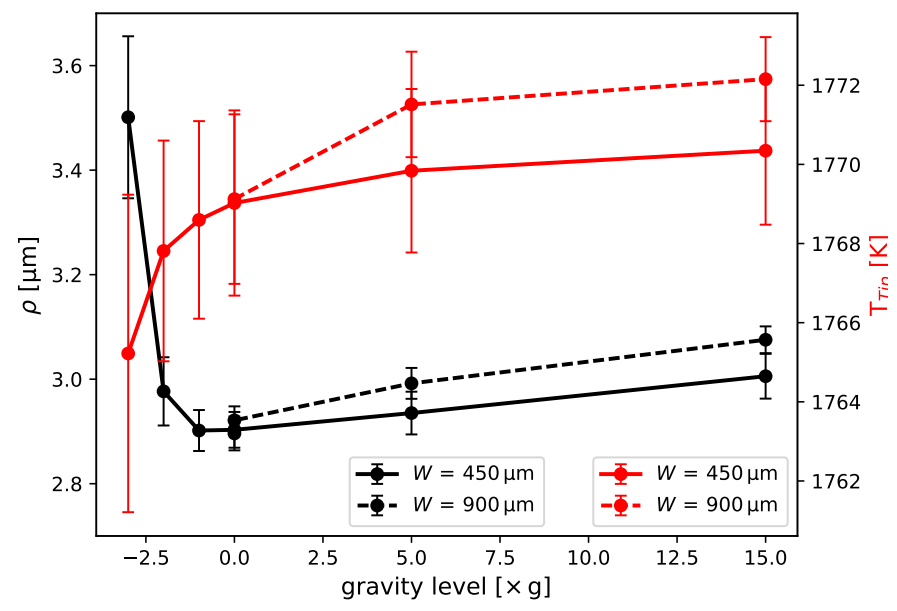

Figure 7: Steady state tip radius $\rho$ and tip temperature $T_{\text {tip }}$ versus gravity level.

To compare our simulations with the empirical law (1), we first show the steady state tip radius $\rho$ and tip temperature $T_{\text {tip }}$ for different gravity levels in Figure 7 . In the gravity range for stable dendritic growth, the tip radius exhibits a minimum around $0 \mathrm{~g}$, with a small influence of the domain width $W$ on the results. The tip temperature increases monotonically with gravity level. The tip radius was calculated by fitting of the tip shape obtained by phase field to a parabola. The length of the box used for the fitting was around 10 times the tip radius. Tip radius values were averaged at steady state ie between 150 to 200 s. From the measurement of tip radii, $\rho^{2} V_{\text {tip }}$ has been calculated and plotted versus the maximum flow velocity, see Figure 8. A linear fit according to Eq. (1) gives $C_{0}=104$ and $\chi^{+}=3000$ for positive gravity levels and $\chi^{-}=10000$ for negative gravity levels, thus for positive gravity levels, the dependency between $\rho^{2} V_{\text {tip }}$ and flow velocity is less pronounced. These values can be compared to $\chi=6400$ and $C_{0}=340$ found by Bouissou [35] for dendritic growth in pivalic acid with downward flow at the tip. Despite the difference between the materials, the same order of magnitude is observed.

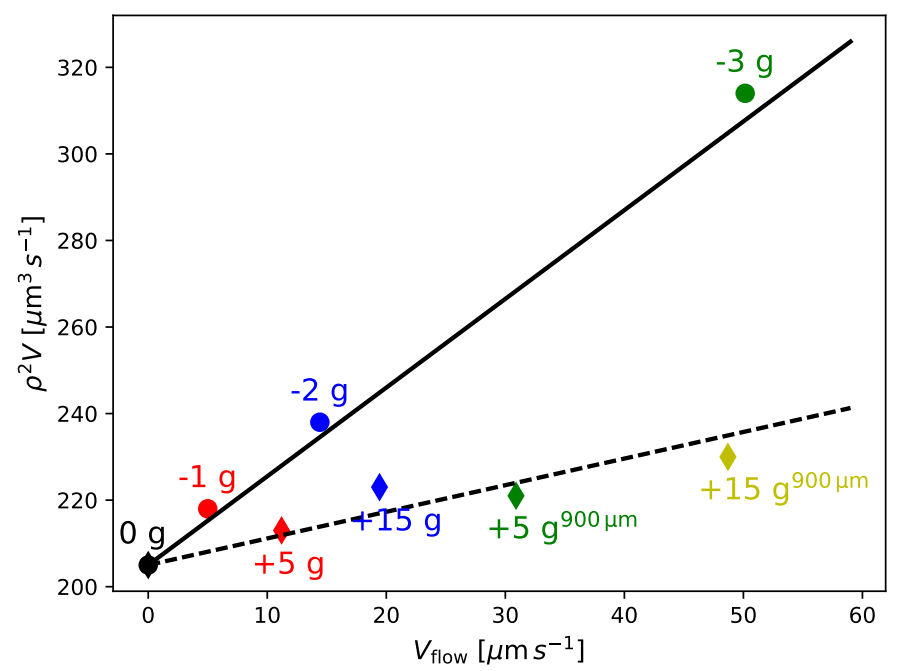

Figure 8: $\rho^{2} V_{\text {tip }}$ versus flow velocity comprising data for different domain sizes.

The dependency of the steady state concentration at the tip, $c_{\text {tip }}$, and the boundary layer thickness $l_{\mathrm{D}}$ are shown in Figure 9. The mass boundary layer thickness is defined here as the vertical distance between the dendrite tip and the iso-contour at $48.6 \mathrm{at} \% \mathrm{Al}$ (white lines in Figure 4). Both, the solute concentration at the tip and $l_{D}$ decreases with increasing gravity level. The flow pattern in Figure 4 showed for all negative gravity levels, that the dendrite tip is fed by aluminum rich interdendritic liquid by the upward flow. As a consequence, the boundary layer thickness increases, too. In contrast, the boundary layer thickness is reduced with downward flow. The impact of flow on the boundary layer thickness is decisive for the formation of branching structures, as we will see in the next section.

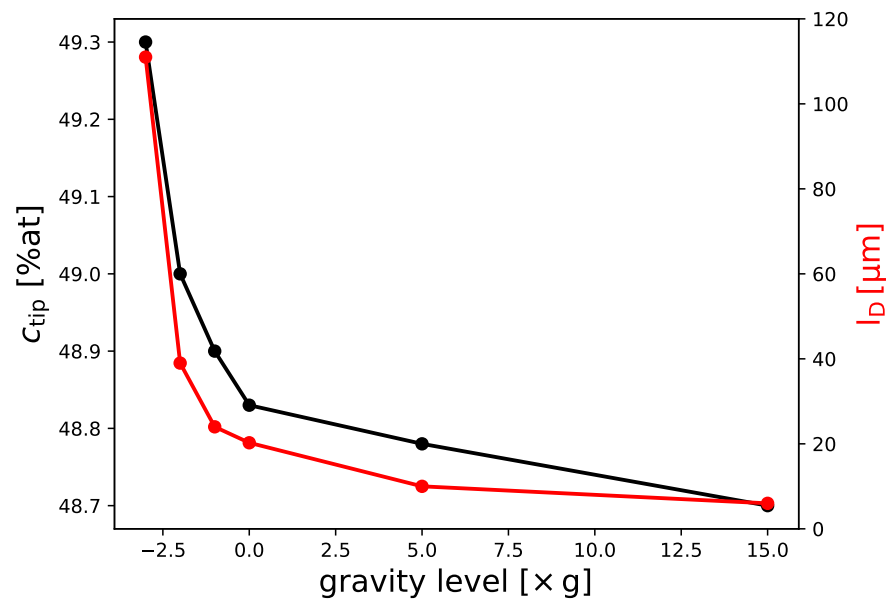

Figure 9: Al-concentration at the tip $c_{\text {tip }}$ and boundary layer thickness $l_{\mathrm{D}}$ versus gravity level. 


\subsubsection{Branched structures}

A major observation is that the stable columnar dendritic growth pattern turns into a highly branched structure when the melt flow pattern becomes unstable. This happens above a critical negative gravity level and depends on the domain size as well. To analyze the mechanisms leading to branched structures, the structure evolution for $-5 g$ and $W=450 \mu \mathrm{m}$ as shown in Figure 4 will be described in more detail. It will be shown that the interplay between the growth of neighboring dendrites and the convection roles in the melt leads to periodic oscillations in the dendritic growth velocity.

In Figure 10, concentration maps superimposed with velocity streamlines are shown for the time interval between $114 \mathrm{~s}$ and $128 \mathrm{~s}$. At the beginning, the left dendrite (1) is growing at a lower tip temperature than dendrite (2) on the right. The tip of dendrite (1) is exposed to a downward flow while the tip of dendrite (2) is exposed to an upward flow. It is important to note that the maximum of the downward flow between the convection rolls is shifted slightly to the right from dendrite (1), thus it is off-axis. In the following, dendrite (1) changes its growth direction, i. e. the growth direction is now tilted against the preferred crystallographic growth direction, towards the maximum of the downward flow stream. At the same time, dendrite (1) accelerates and eventually grows ahead of dendrite (2). Between $120 \mathrm{~s}$ and $122 \mathrm{~s}$, the flow pattern changes in response to the change of the dendrite arrangement and the increasing $\mathrm{Al}$ segregation ahead of the accelerating dendrite (1). At around $124 \mathrm{~s}$, the convection rolls have been rearranged and the tip of dendrite (1) is exposed to an upward flow, showing a decreasing growth velocity accompanied by a tip splitting event.

Now, dendrite (2) is taking the role of dendrite (1) and a similar processes takes place in a periodic manner. This can be seen in Figure 11. The evolution of the tip velocity $\mathrm{V}_{\text {tip }}$, tip radius $\rho$ (measured with the same method as in section 4.2 but at every second without averaging and when the tip remains parabolic), boundary layer thickness $l_{\mathrm{D}}^{*}$ and the flow velocity in $z$-direction at the tip, $\mathrm{V}_{\text {flow }}$, are plotted for a time interval ranging from $110 \mathrm{~s}$ to $170 \mathrm{~s}$. The $\mathrm{V}_{\text {tip }}$ curves show that the two tip velocities are outof-phase and oscillate around the pulling speed. The tip velocity is linked to the boundary layer thickness, when the boundary layer thickness is small, i.e. a steep concentration gradient, the tip velocity is large and reciprocally. The boundary layer thickness is also affected by the melt flow. When the flow velocity is negative, i.e. downward flow on tip, $l_{D}$ is smaller and when the flow velocity is positive, i.e. upward flow, $\mathrm{l}_{\mathrm{D}}$ is larger. The curves of the measured tip radii $\rho$ are not continuous because they were only measured before tip destabilization and splitting. The tip radius itself is phase shifted to the tip velocity, when velocity is high, the tip radius is low and reciprocally. The period of the oscillations is approximately $30 \mathrm{~s}$.

Chen et al. [40] have proposed a scaling law for the tip splitting frequency $\tau_{\mathrm{i}}$ and applied it to an $\mathrm{AlCu} 4 \mathrm{wt} \%$ alloy. They have found: $\tau_{\mathrm{i}}=A D_{\mathrm{l}} / V_{\text {pull }}^{2}$ with a constant prefactor $A$ ranging from 0.3 to 3 . Our results lead to $A \approx 6$ which is in the same order of magnitude, however, here the tip splitting appears along a quasi-stationary process and is related to the interplay with gravity induced melt flow compared to the purely diffusive, but transient growth conditions investigated in [40].

The results for higher negative gravity levels $(<-5 g)$ are not periodic anymore but the following trends can be identified:

- The PDAS selection is already affected by the melt flow at an early stage of the solidification process, i.e. directly after the onset of the first interface instabilities, see Figure 5.

- At a later stage, instationary flow pattern leads to oscillations of the dendrite tip velocities $V_{\text {tip }}$ around the pulling velocity $V_{\text {pull }}$. Consequently, the tip radii oscillates as well. These oscillations may trigger tip splitting events.

- The number of temporal changes of the growth direction and of corresponding tip splitting and branching events is increasing with increasing gravity levels and flow velocities, respectively, see Figure 6.

- The branching process comprises (i) a change of the apparent dendrite growth direction and (ii) the realignment of the growth direction with the preferential crystallographic direction.

The evolution of a single dendrite tip along a branching process is detailed in figure 12 by a cut out from the tip region of dendrite (1) in Figure 10. In the first three pictures, the dendrite growth direction is aligned with the crystallographic preferred direction, but the isoconcentration lines are not symmetric around the dendrite tip, instead they are tilted to the right caused by the downward melt flow which is also off centered to the dendrite axis. Beginning at $\mathrm{t}=114 \mathrm{~s}$, the growing tip responds to the concentration field and changes its growth direction towards the steepest gradient (smallest distance of the isolines) in the concentration field which is tilted to the vertical axis, pointing towards the largest downward flow velocities. At the same time, the tip velocity increases (compare Figure 10) because the concentration gradient gets steeper and $V_{t i p} \sim \nabla c$. Under pure diffusive growth conditions, small modifications of the symmetry of the solutal profile caused by the superposition of the diffusion fields of neighboring dendrites may lead to a slow sideward movement of individual dendrites, called "phase diffusion" [41], which evens out the dendrite spacings. Compared to this, the flow induced change of the tip trajectory is an extreme event and leads to a faster adjustment of the dendrite spacings.

At around $119 \mathrm{~s}$, the flow pattern starts to respond to the modified growth configuration and the convection 


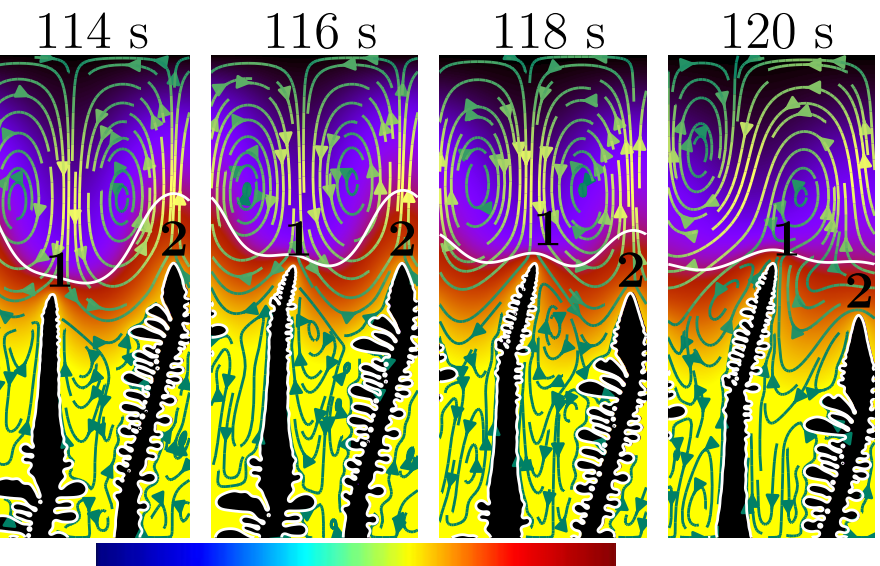

48 Liquid concentration $\mathrm{Al}$ [\% at] 49.4
$122 \mathrm{~s}$
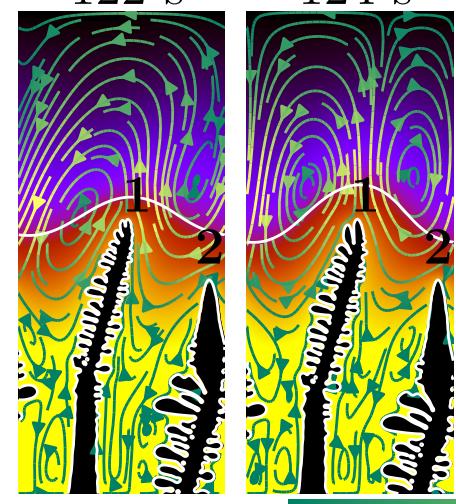

Melt velocity $\left[\mathrm{ms}^{-1}\right]$
$128 \mathrm{~s}$

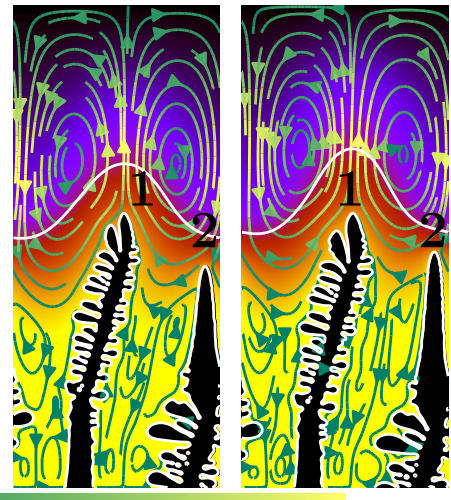

$V_{\max }$

Figure 10: Temporal evolution of the concentration field superimposed with velocity streamlines between $114 \mathrm{~s}$ and $128 \mathrm{~s}$ (domain width $W=450 \mu \mathrm{m}$, gravity level $-5 \mathrm{~g})$. The white line visualizes the isoline for $48.6 \mathrm{at} \% \mathrm{Al}$.

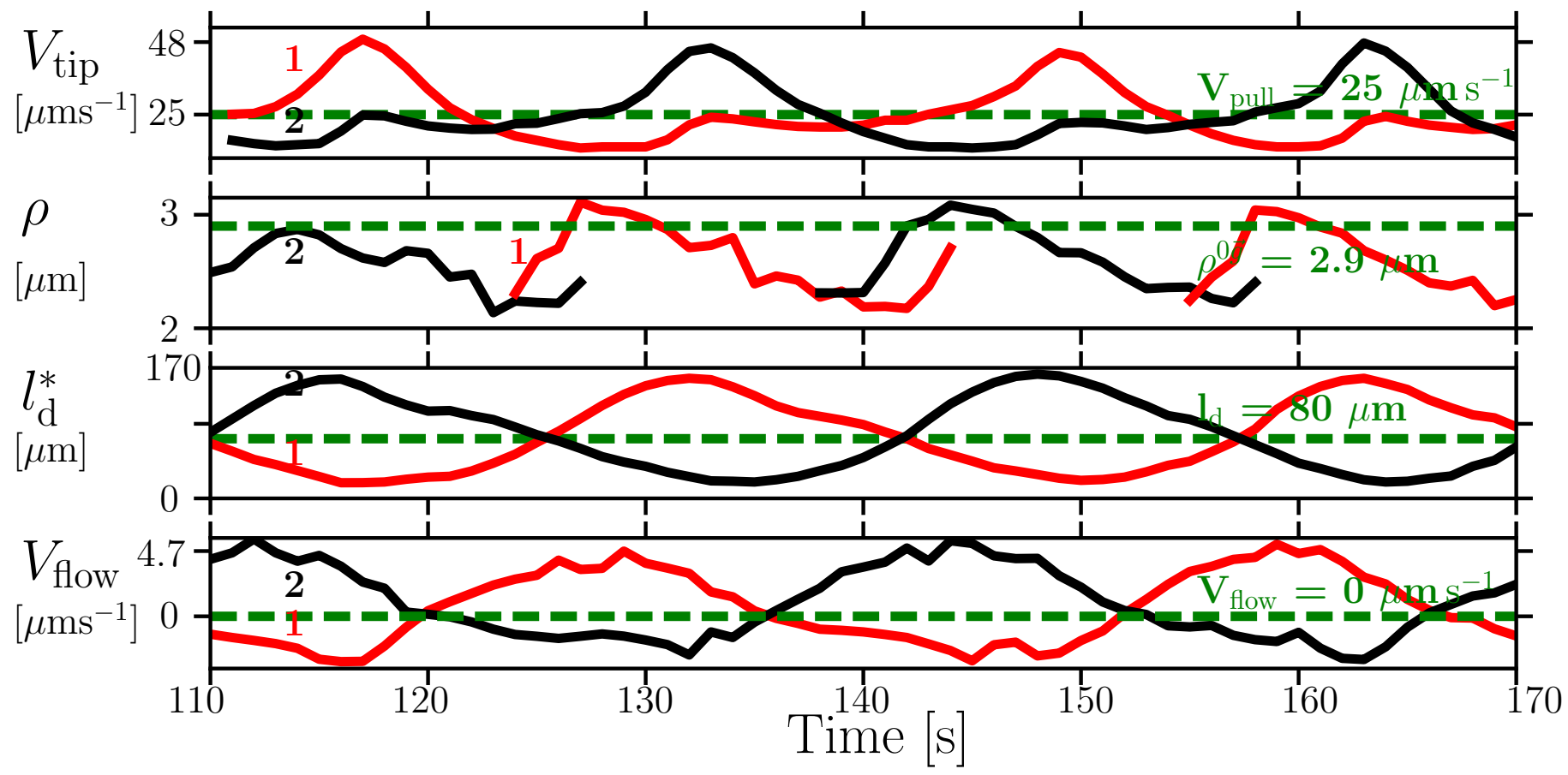

Figure 11: Evolution of the tip radius, tip velocity, boundary layer thickness and flow velocity at the tip. The red and black curves correspond to the dendrites marked 1 and 2 on Figure 10, respectively. 
rolls reorganize in a way that the local flow around the tip changes from downward to upward flow. Correspondingly, the concentration gradient becomes smaller, the tip velocity decreases and the tip radius increases. At this point, the dendrite tip exhibits an intermediate instability resulting in a tip splitting event. Both tips continue to grow, now again along the preferred crystallographic orientation because the impact of the melt flow on the symmetry of the concentration field is reduced, i.e. the isolines are almost symmetric and parabolic around the vertical axis. At $123 \mathrm{~s}$, two tips are well developed but later on, a single tip becomes stable again, growing along the symmetry plane of the upward flow between two convection roles.

In a nutshell, variations in the growth direction and tip splitting are induced by the interplay between the flow pattern and the dendritic growth dynamic. Resulting deviations from an Ivantsov type growth behavior can be interpreted as flow induced anisotropy caused by the perturbations of the local concentration field around the dendrite tip which affects the local growth velocity of the solidliquid interface. This effect has been already observed in phase-field simulations for equiaxed growth with flow [42]. Supplementary simulations (not presented here) have shown that with a stiffness anisotropy twice higher, the splitting effects are suppressed. This means that the tendency for tip splitting and branching is material dependent. How sensitive the dendritic growth structure responds on melt flow depends on the alloy as well. The dendritic growth morphologies inferred from metallographic pictures at least suggest the conclusion that the anisotropy of the bcc $\beta$-Ti/melt interface is rather weak.

Any impact of melt flow on the solidification morphologies, either on the shape and dynamics of individual dendrites or on the PDAS selection in dendritic arrays becomes more likely for a larger ratio of the solute diffusion layer over the hydrodynamical boundary layer. The results in this paper have been achieved for $D / V_{\text {tip }}=120 \mu \mathrm{m}$, the results by Steinbach [13] on Al-Cu for $D / V_{\text {tip }}=75 \mu \mathrm{m}$ and those observed by Bataille [16] on Pb-Sn with $D / V_{\text {tip }}=34 \mu \mathrm{m}$ In all three examples, the PDAS changes at higher gravity levels. Recently, Zimmermann et al. published experiments on the effect of microgravity and hypergravity on Neopentylglycol-(D)Camphor, an organic and transparent alloy [43]. No PDAS changes was observed even at higher gravity and flow velocities close to the ones in our TiAl simulations, i.e. up to a few hundred of micrometers per seconds. This discrepancy can be explained by the much smaller diffusion layer thickness, and therefore much weaker perturbation of the solutal field by flow, of about $4 \mu \mathrm{m}$ which is at least one order of magnitude lower than in this work, Steinbach [13] and Bataille [16].

\section{Conclusion}

The phase-field method was applied to simulate the evolution of solidification microstructures of Ti48 at $\% \mathrm{Al}$ during directional solidification including buoyancy driven melt flow under different gravity levels. In similar studies so far, the major aspect was to investigate the effect of melt flow on the primary dendrite spacing with the general behavior that upward flow reduces the spacing and downward flow leads to an increase of the spacing. The results in this paper are focused on the operating state of the dendrite tips, e.g. tip undercooling and its stability against tip splitting or changes of the apparent growth direction.

On one hand, in the case of positive gravity, a stable configuration with symmetric convection rolls can be observed with downward flow around the dendrite tips. Even for high values of positive gravity $(15 \mathrm{~g})$, the maximal flow velocity does not significantly exceed the pulling velocity of $25 \mu \mathrm{m} / \mathrm{s}$. On the other hand, if the gravity value is negative, the dendrite tips are exposed to upward flow and the dendritic growth pattern destabilises when the flow velocity reaches a critical value of twice the pulling velocity. At least for smaller anisotropies of the interfacial stiffness, tip splitting can occur. In addition, dendrites may change their growth direction, not growing along the preferential crystallographic direction but following the steepest chemical gradient in the flow affected concentration field. This can be also considered as an alternative mechanism for the adjustment of the primary dendrite arm spacing, in addition to tertiary arm formation and phase diffusion.

If the melt convection is comparable to the diffusion fluxes, dynamical changes in the flow pattern prevents stable parabolic, Ivantsov-type concentration isolines around the dendrite tips. As a consequence, a dynamic interchange between flow and growth pattern is observed which can also result in quasi-periodic oscillations in the operating state of neighboring dendrites linked to periodic changes of the rotational direction of melt flow vortices.

An investigation with three-dimensional simulations should be done, where the influence of dimensionality, domain size and gravity levels are to be studied in details. A highly branched microstructure may have an impact on the fragmentation of dendrite arms and thus enhance the number of equiaxed grains during CET. This requires simulations linking flow, microstructure and mechanical forces to further elucidate the correlation between solidification morphology and fragmentation [44].

\section{Acknowledgment}

The authors would like to thank Neil Melville, Antonio Verga, Jack van Loon at ESA and Burkhard Schmitz group at Airbus Defense Space. The authors also thank Benoit Appolaire, Miha Založnik, Hervé Combeau, Gerhard Zimmermann and Guillaume Boussinot for fruitful discussions. This work was carried out as part of the GRADECET (GRAvity DEpendence of Columnar to Equiaxed Transition in TiAl Alloys) research project. We gratefully acknowledge the support by the German Federal Ministry of Research through the German Space Agency DLR under contract FKZ 50WM1743. 

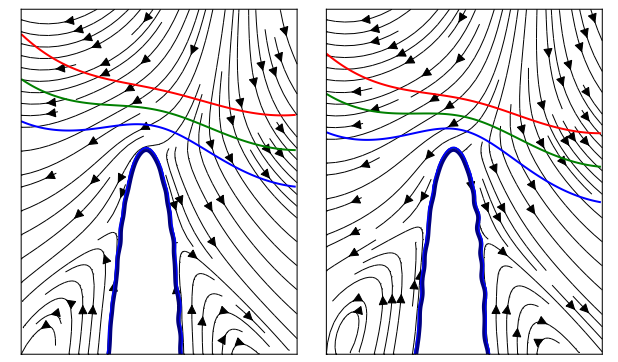

$111 \mathrm{~s}$

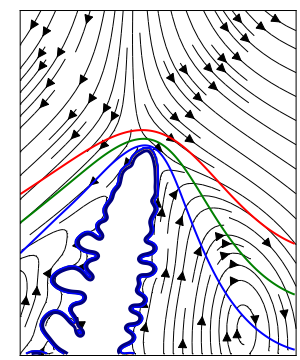

$119 \mathrm{~s}$

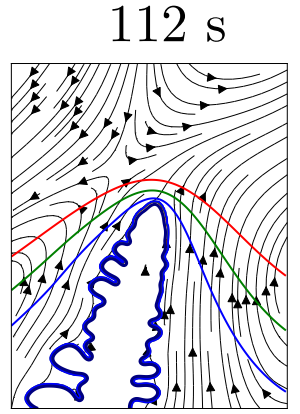

$120 \mathrm{~s}$

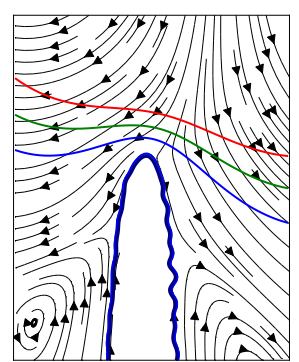

$113 \mathrm{~s}$

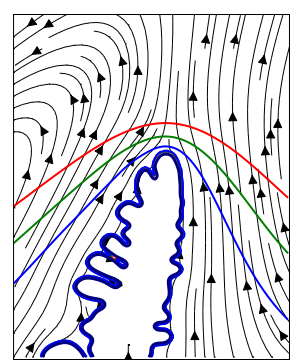

$121 \mathrm{~s}$

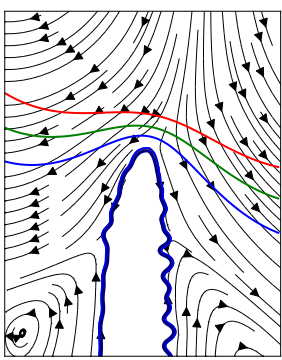

$114 \mathrm{~s}$

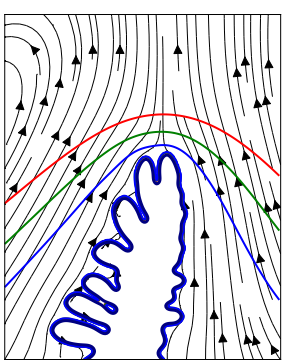

$122 \mathrm{~s}$

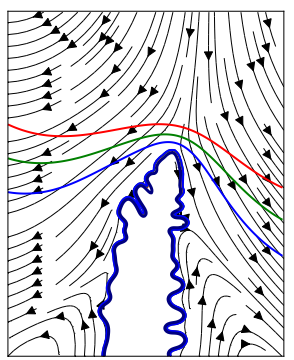

$115 \mathrm{~s}$

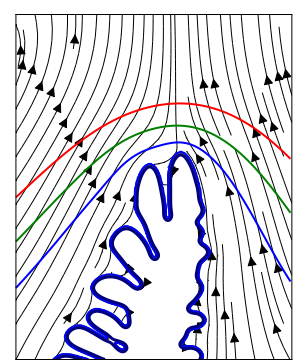

$123 \mathrm{~s}$

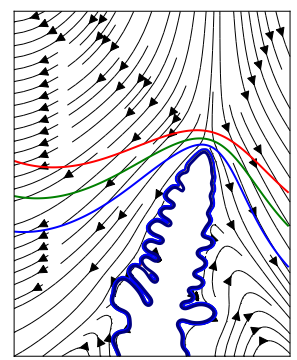

$116 \mathrm{~s}$

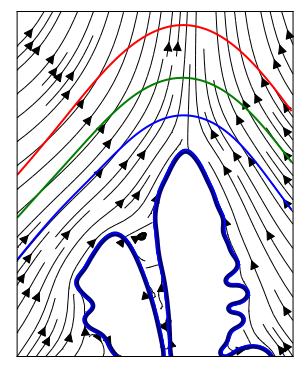

$130 \mathrm{~s}$

Figure 12: Evolution of a dendrite tip superimposed with the flow pattern, concentration isolines are given for 48.8 (red), 48.9 (green), 49 (blue) at\% Al. Gravity level: $-5 \mathrm{~g}$, domain width: $\mathrm{W}=450 \mu \mathrm{m}$, the size of the selected cut out is $150 \times 187.5 \mu \mathrm{m}^{2}$.

\section{References}

[1] G Zimmermann, L Sturz, B Billia, N Mangelinck-Noel, H Nguyen Thi, ChA Gandin, D J Browne, and D J Mirihanage. Investigation of columnar-to-equiaxed transition in solidification processing of alsi alloys in microgravity - the cetsol project. J. Phys.: Conf. Ser., 327:012003, 2011.

[2] M Becker, S Klein, and Kargl F. Free dendritic tip growth velocities measured in al-ge. Phys. Rev. Mat., 2:073405, 2018.

[3] M E Glicksman, Koss M B, Bushnell L T, LaCombe J C, and Winsa E A. Space flight data from the isothermal dendritic growth experiment. Adv. Space Res., 16(7):181-184, 1995.

[4] M E Glicksman, Koss M B, Bushnell L T, LaCombe J C, and Winsa E A. Dendritic growth of succinonitrile in terrestrial and microgravity conditions as a test of theory. Iron and steel institute of japan international, 35(6):604-610, 1995.

[5] W J Boettinger, J A Warren, C Beckermann, and Karma A. Phase-field simulation of solidification. Annu. Rev. Mater. Res., 32:163-194, 2002

[6] W U Mirihanage, D J Browne, G Zimmermann, and L Sturz. Simulation of international space station microgravity directional solidification experiments on columnar-to-equiaxed transition. Acta Mater., 60:6362-6371, 2012

[7] D R Liu, N Mangelinck-Noel, Ch-A Gandin, G Zimmermann, L Sturz, H Nguyen-Thi, and B Billia. Simulation of directional solidification of refined al-7 wt.\%si alloys - comparison with benchmark microgravity experiments. Acta Mater., 93:24-37, 2015.

[8] S Boden, S Eckert, B Willers, and Gerbeth G. X-ray radioscopic visualization of the solutal convection during solidification of a ga-30 wt pct in alloy. Metall. Mater. Trans. A, 39A:613-623, 2008.

[9] C Beckermann and R Viskanta. Natural convection solid/liquid phase fhange in porous media. Int. J. Heat Mass Trans. 31(1):35-46, 1988.

[10] L Yuan and P D Lee. Microstructural simulations of the influence of solidification velocity on freckle initiation during directional solidification. ISIJ International, 50:1814-1818, 2010.

[11] Y Chen, H Nguyen-Thi, D Z Li, A A Bogno, B Billia, and N M Xiao. Influence of natural convection on microstructure evolution during the initial solidification transient: comparison of phase-field modeling with in situ synchrotron x-ray monitoring data. IOP Conf. Ser.: Mater. Sci. Eng., 33:012102, 2012.

[12] T Takaki, $\mathrm{R}$ Rojas, $\mathrm{S}$ Sakane, M Ohno, $\mathrm{Y}$ Shibuta, $\mathrm{T}$ Shimokawabe, and $\mathrm{T}$ Aoki. Phase-field-lattice boltzmann studies for dendritic growth with natural convection. J. Cryst. Growth, 474:146-153, 2017.

[13] I Steinbach. Pattern formation in constrained dendritic growth with solutal buoyancy. Acta Mater., 57:2640-2645, 2009.

14] M Apel and H J Diepers. On the effect of interdendritic flow on primary dendrite spacing: a phase field study and analytical scaling relations. MCWASP XI Proceedings, pages 505-512, 2006.

[15] H J Diepers and I Steinbach. Interaction of interdendritic convection and dendritic primary spacing: phase-field simulation and analytical modeling. Material Science Forum, 508:145-150, 2006

[16] C C Bataille, R N Grugel, A B Hmelo, and T G Wang. The effect of enhanced gravity levels on microstructural development in pb-50 wt pct sn alloys during controlled directional solidification. Metal Trans A, 25:865-870, 1994.

[17] J Aguilar, A Schievenbusch, and O Kättlitz. Investment casting technology for production of tial low pressure turbine bladesprocess engineering and parameter analysis. Intermetallics, 19(6):757-761, 2011.

[18] P X Fu, X H Kang, Y C Ma, K Liu, D Z Li, and YY Li. Centrifugal casting of tial exhaust valves. Intermetallics, 16(2):130-138, 2008.

[19] B P Bewlay, M Weimer, T Kelly, A Suzuki, and P R Subramanian. The science, technology, and implementation of tial alloys in commercial aircraft engines. MRS Online Proceedings Library Archive, 1516:49-58, 2013.

[20] J Zollinger, A Viardin, D Daloz, C Huang, and U Hecht. Columnar dendritic growth of ti-48al-2cr-2nb alloy during directional solidification in micro- and hyper-gravity. To be published.

[21] J J W A van Loon, J Krausse, and H Cunha. The large diameter centrifuge, ldc, for life and physical sciences and technology. Proceedings of the "Life in Space for life on earth symposium", 2008.

[22] M Li, T Mori, and H Iwasaki. Effect of solute convection on the primary arm spacings of pb-sn binary alloys during upward directional solidification. Materials Science and Engineering : 
A, 265:217-223, 1999 .

[23] Micress version 6.1. 2014.

[24] J Eiken, B Bottger, and I Steinbach. Multiphase-field approach for multicomponent alloys with extrapolation scheme for numerical application. Phys. Rev. E, 73:066122, 2006.

[25] C Carré, B Böttger, and A Apel. Implementation of an antitrapping current for a multicomponent multiphase-field approach. $J$. Cryst. Growth, 380:5-13, 2013.

[26] B Echebarria, R Folch, and M Plapp. Quantitative phase-field model of alloy solidification. Phys. Rev. E, 70:061604, 2004.

[27] J Eiken. Numerical solution of the phase-field equation with minimized discretization error. IOP Conference Series Materials Science and Engineering, 33:012105, 2012.

[28] C Beckermann, H J Diepers, I Steinbach, A Karma, and X Tong. Modeling melt convection in phase-field simulations of solidification. J. Comp. Phys., 154:468-496, 1999.

[29] Y Lu, C. Beckermann, and J C Ramirez. Three-dimensional phase-field simulations of the effect of convection on free dendritic growth. J. Crys. Growth, 280:320-334, 2005.

[30] J-H Jeong, N Goldenfeld, and J Dantzig. Phase field model for three-dimensional dendritic growth with flow. Phys. Rev. E, 64:041602, 2001.

[31] J-H Jeong, J Dantzig, and N Goldenfeld. Dendritic growth with fluid flow in pure materials. Met. Mat. Trans. A, 34:459-466, 2003.

[32] S Battaglioli, AJ Robinson, and S McFadden. Influence of natural and forced gravity conditions during directional columnar solidification. Int. J. Heat Mass Tran., 126:66-80, 2018.

[33] M Ben Amar and Y Pomeau. Needle-crystal growth in an axial flow - scaling laws. Comptes rendus de l'Academie des sciences, Serie 2, 308:907-914, 1989.

[34] P Bouissou and Y Pelcé. Effect of a forced flow on dendritic growth. Phys. Rev. A, 40:6673, 1989.

[35] $\mathrm{P}$ Bouissou. Influence d'un écoulement sur la croissance dendritique : Aspect Théorique et Expérimental. PhD thesis, Ecole Normale Supérieure, 1989.

[36] J Maurer, P Bouissou, B Perrin, and P Tabeling. Faceted dendrites in the growth of nh4br crystals. Europhysics Letters, 8:67, 1989.

[37] V Emsellem and P Tabeling. Experimental study of dendritic growth with an external flow. J. Crys. growth, 156:285-295, 1995.

[38] P Bouissou, B Perrin, V Emsellem, and P Tabeling. Growth and Forms. Plenum Press, New York, 1991.

[39] Y W Lee, R Ananth, and W N Gill. Selection of a length scale in unconstrained dendritic growth with convection in the melt. J. Crys. growth, 132:226-230, 1993.

[40] Y Chen, B Billia, D-Z Li, H Nguyen-Thi, N-M Xiao, and AA Bogno. Tip-splitting instability and transition to seaweed growth during alloy solidification in anisotropically preferred growth direction. Acta Mater., 66:219-231, 2014.

[41] Y Song, S Akamatsu, S Bottin-Rousseau, and Karma A. Propagative selection of tilted array patterns in directional solidification. Phys. Rev. Materials, 2:053403, 2018.

[42] Z Guo, J Mi, S Xiong, and P S Grant. Phase field simulation of binary alloy dendrite growth under thermal- and forced- flow fields: An implementation of the parallel-multigrid approach. Metall. Mater. Trans. B, 44B:924-937, 2013.

[43] G Zimmermann, Hamacher M., and Sturz. Effect of zero, normal and hyper-gravity on columnar dendritic solidification amd the columnar-to-equiaxed transition in neopentylglycol(d)camphor alloy. J. Cryst. Growth: Conf. Ser., 512:47-60, 2019.

[44] H Neumann-Heyme, K Eckert, and C Beckermann. Dendrite fragmentation in alloy solidification due to sidearm pinch-off. Phys. Rev. E, 92:060401, 2015.

[45] A S Lopis. Computational simulation of molten titaniumaluminum metal and alloys. 2010.

[46] R K Wunderlich. Surface tension and viscosity of industrial ti-alloys measured by the oscillating drop method on board parabolic flights. High Temp. Mat. and Proc., 27:401-412, 2008.
[47] V T Witusiewicz, A Bondar, U Hecht, S Rex, and T Ya. The Al-B-Nb-Ti system I. Re-assessment of the constituent binary systems B-Nb and B-Ti on the basis of new experimental data. J. Alloy Compd, 448:185-194, 2008.

[48] K Binder and F Kargl. Private communication, 2019.

[49] J Eiken, M Apel, V T Witusiewicz, J Zollinger, and U Hecht. Interplay between $\alpha(\mathrm{ti})$ nucleation and growth during peritectic solidification investigated by phase-field simulations. J. Phys.: Cond.Mat.21, page 464104, 2009. 